\title{
Primary peritoneal serous papillary carcinoma (PSPC) involving ovary and colon: Management and Treatment
}

\author{
Leanza $\mathrm{V}^{1}$, , Coco $\mathrm{L}^{1}$, Leanza $\mathrm{G}^{1}$, Scilletta $\mathrm{B}^{1}$, Zanghì $\mathrm{G}^{1}$, Vecchio $\mathrm{R}^{1}$ and Zarbo $\mathrm{G}^{1}$ \\ ${ }^{1}$ Surgery Department, Catania University, Italy
}

\begin{abstract}
We present a case report of a 47-year-old woman who was admitted to our University-Hospital following diagnosis of pelvic mass. Abdominal examination revealed a tender, palpable mass on the right iliac region. At the gynecological examination uterus was regular in size. On the left side of the uterus a mass of $9 \mathrm{~cm}$ was observed; its surface was irregular and no mobility was found. Abdominal CT and NMR revealed massive ascites, omental cake and increased volume of both ovaries. Patient underwent longitudinal suprombelical-pubic laparotomy. After opening abdominal cavity, a free-fluid sample was taken and the results were positive for malignant cells. Typical neoplastic localizations on both ovaries, Douglas' peritoneum, rectum, sigmoid colon and omentum were observed. Extemporaneous histological examination diagnosed a peritoneal serous papillary carcinoma. Hysterectomy with salpingo oophorectomy, total omentectomy, appendectomy, pelvic and lumbo-aortic lymphadenectomy was performed. Retroperitoneal approach to remove the whole Douglas' peritoneum together with the pouch malignant localizations was done. Sigmoid colon and rectum were resected. A latero-terminal anastomosis with stapler was performed. All the visible abdominal maligant lesions were cut out. No transfusion was necessary. The postoperative course was regular and after seven days the patient was discharged. Chemotherapy ended the therapeutic management (six cycles of carboplatin| with paclitaxel)|. After one year the patient is in good health and instrumental investigations (Ultrasounds, TC and NMR) are negative for recurrence. Such a case is very interesting for the discrepancy between slight symptoms and severity of the disease, the solution of which was very complex requiring a skillful polyspecialized oncological team.
\end{abstract}

Keywords: papillary carcinoma; pelvic mass; chemotherapy

\section{Background}

Primary peritoneal serous papillary carcinoma (PSPC) is a rare tumor of the peritoneum affecting mainly elderly and post-menopausal women. Histologically it is similar to serous papillary carcinoma of the ovary. The epithelial layer of the ovary and the peritoneum share a common embryonic heritage, deriving from coelemic epithelium [1].

PSPC presents as peritoneal carcinomatosis without evident tumoral focus. Although the pathogenesis of PSCP remains unclear, genealogical studies have led to the inclusion of peritoneal carcinoma in the hereditary breast ovarian cancer (HBOC) syndrome, which also includes fallopian tube neoplasms. Genotype analysis revealed a partial correlation between primary peritoneal carcinoma and BRCA mutations (185delAG and 5382insC in BRCA1 and 6174delT in BRCA2) [2, 3]. In 1993, the Gynecologic Oncology Group developed specific diagnostic criteria allowing the differentiation of PSCP from papillary serous ovarian carcinoma [4], which has similar histological aspect but different prognosis [5]. Ovarian serous tumors are generally discovered after spreading into the peritoneal surfaces; they are arbitrarily assigned to the ovary when involvement of this organ overtakes $5 \mathrm{~mm}$ [6]. This observation supports the hypothesis that papillary serous ovarian carcinoma originates from the epithelium

*Corresponding author: Leanza V, Surgery Department, Catania University, Italy, Email: vito.leanza@gmail.com

Received 30 November 2012 Revised 16 April 2013 Accepted 23 April 2013 Published 30 April 2013

Citation: Leanza V, Coco L, Leanza G, Scilletta B, Zanchì G, Vecchio R, Zarbo G (2013) Primary peritoneal serous papillary carcinoma (PSPC) involving ovary and colon: Management and Treatment. J Cancer Res Ther 1: 119-122. doi:10.14312/2052-4994.2013-18

Copyright: (C)2013 Leanza V, et al. This is an open-access article distributed under the terms of the Creative Commons Attribution License, which permits unrestricted use, distribution and reproduction in any medium, provided the original author and source are credited. 
of the peritoneal cavity and secondary involves the ovarian tissue $[7,8]$.

\section{Case presentation}

We present a case report of a 47-year-old woman who was admitted to our University-Hospital following diagnosis of pelvic mass. She suffered from gastrointestinal symptomatology, observing alteration of bowel habit consisting in constipation alternated with diarrhea and associated with discomfort on the left iliac region. Abdominal examination revealed a tender, palpable mass on the right iliac region. At the gynecological examination uterus was regular in size. On the left side of the uterus a mass of $9 \mathrm{~cm}$ was observed; its surface was irregular and no mobility was found. The mass was painful during palpation and it compressed both sigmoid colon and rectum. Right ovary was regular in size and fix.

Initial routine blood tests showed $14.97 \mathrm{~g} / \mathrm{dl}$ of hemoglobin, normal mean cell volume and no abnormalities of electrolytes, liver or kidney function were observed. Tumor markers revealed an increase of CA125:47,02 U/ml and CA 15-3: 61,15 U/ml, whereas CA 19-9 was normal ( $3 \mathrm{U} / \mathrm{ml}$ ). Abdominal CT and NMR revealed massive ascites, omental cake and increased volume of both ovaries.

Patient underwent longitudinal suprombelical-pubic laparotomy. After opening abdominal cavity, a freefluid sample was taken and the results were positive for malignant cells. Typical neoplastic localizations on both ovaries, Douglas' peritoneum, rectum, sigmoid colon and omentum were observed.

Extemporaneous histological examination diagnosed a peritoneal serous papillary carcinoma. Hysterectomy with salpingo oophorectomy (Figure 1), total omentectomy, appendectomy, pelvic and lumbo-aortic lymphadenectomy were performed. Surgeons chose retroperitoneal approach in order to remove the Douglas' peritoneum together with the pouch malignant localizations.

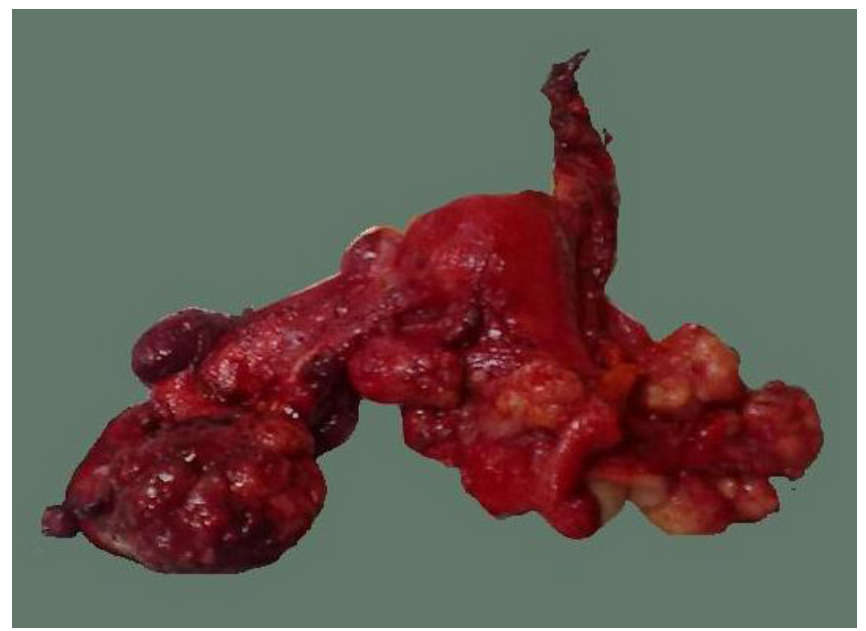

Figure 1 Uterus without the left adnexum (the right ovary was sent before for the histological examination)
Sigmoid colon and rectum were resected (Figure 2). A latero-terminal anastomosis with stapler was performed (Figure 2). After opening the rectum a crateriform lesion was observed (Figure 3). Finally, all the visible abdominal maligant lesions were cut out. No transfusion was necessary. The postoperative course was regular and after seven days the patient was discharged. Final histological examination was not able to establish if the ovarian lesions were primary or secondary to a peritoneal extra ovarian serous carcinoma. Chemotherapy ended the therapeutic management (six cycles of carboplatin and paclitaxel). After one year the patient is in good health and instrumental investigations (Ultrasounds, TC and NMR) are negative for recurrence.

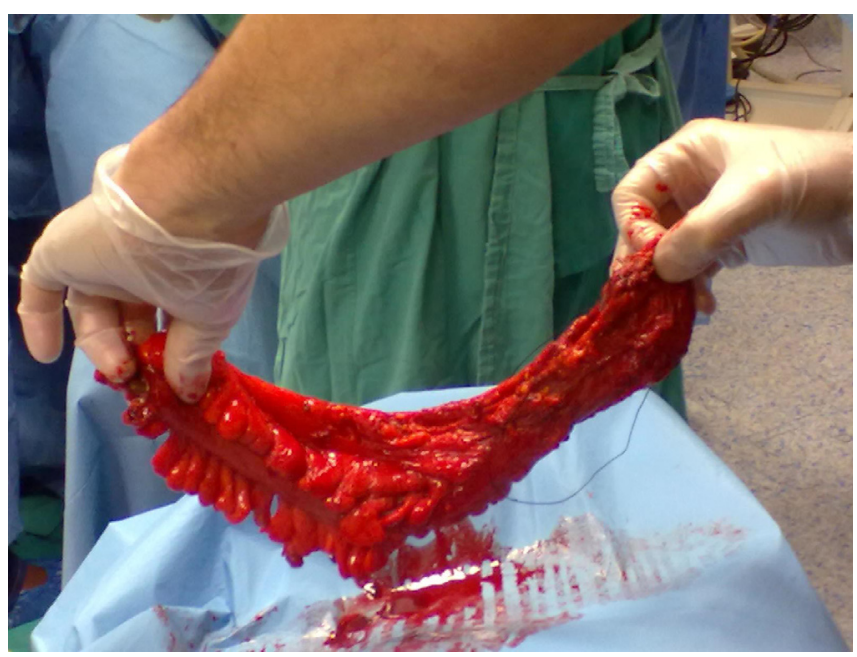

Figure 2 Surgical resection of the sigmid colon and rectum

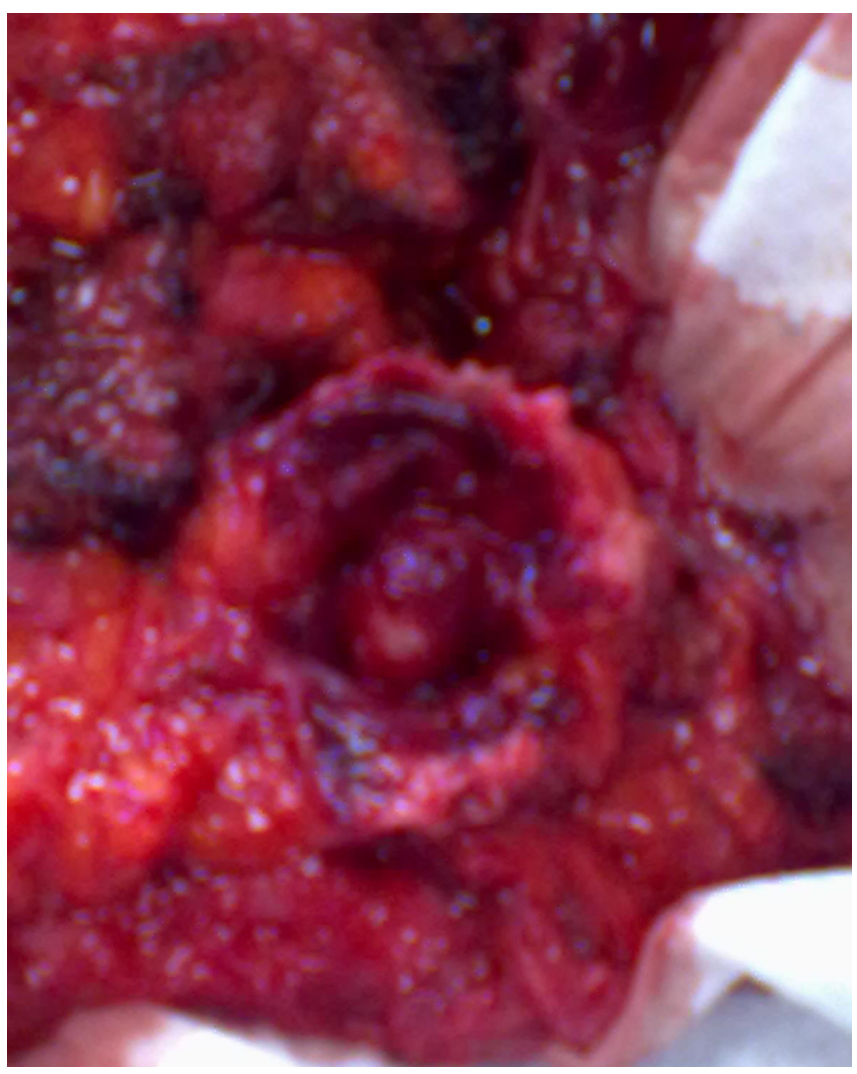

Figure 3 Crateriform lesion of the rectum 


\section{Discussion}

Management of PSPC is similar to ovaric neoplasms, although prognosis should be worse. Long-term survival has been described with cytoreductive surgery and adjuvant chemotherapy with platinum. Surveillance remains the best course of management for patients with known BRCA gene mutations, particularly.

Similar to the epithelial ovarian cancer [9], a high percentage of women with PSCP were diagnosed in an advanced stage, in spite of the aggressive nature of this lesion.

PSCP is diagnosed in the presence of a large, generally disseminated, tumor mass involving peritoneal surfaces with minimal or nonexistent tubal and ovarian involvement [10, 12]. High-grade serous carcinomas, characterized by genetic instability and TP53 mutations, are thought to arise in the fallopian tube mucosa or the mullerian epithelium involving the peritoneum and the ovary secondarly $[7,8]$.

The tubal fimbria, which is close to the ovarian surface and exposed to the peritoneal cavity, has been identified as one of the most common sites of early serous carcinoma for BRCA-positive women [11].

\section{Conclusion}

This case report offers the topic of some consideration as follows: (1) Difficulty of diagnosis, (2) Surgical treatment, (3) Adjuvant chemotherapy.

As for as the clinic diagnosis, initial peritoneal cancer does not give specific symptoms and, accordingly, it is hard to detect in the early stages. That's because its symptomatology is vague. When clear symptoms occur, the disease has progressed. Then, symptoms resemble those of ovarian cancer. Many of these symptoms are a consequence of fluid increase (ascites) in abdominal cavity. Peritoneal cancer symptoms may include:

- Abdominal discomfort or pain from gas, indigestion, pressure, swelling, bloating or cramps

- Feeling of fullness, even after a light meal

- Nausea or diarrhea

- Constipation

- Pollakiuria

- Loss of appetite and weight loss

- Abnormal vaginal or rectal bleeding.

PSPC is often detected when lesion involves many organs. In such cases, it is difficult to detect the true origin even when sophisticated imaging techniques (US, TC, NMR) are employed. Besides, final histological examination may be not able to establish if the lesions of peritoneal sheet are either primary or secondary, as it happened in this case report.
Regarding thesurgical treatment, the operation plays a very important role and affects the prognosis predominantly. During surgery, the removal of all malignant lesions offers a better life expectancy. Collaboration between gynecological and surgical team is mandatory.

The operation needs a very great effort. Regarding the specific technique we must emphasize that retroperitoneal approach offers the advantage of removing the parietal peritoneum together with the intra-abdominal lesions; nevertheless its execution is challenging and requires surgical expertise. As regards the adjuvant chemotherapy, it is important for the whole management of disease. Chemotherapy employs anti-cancer (cytotoxic) drugs to destroy malignant cells. Both PSPC and ovarian cancer are usually very sensitive to chemotherapy. Chemotherapy is usually given after surgery to reduce the risk of the malignant recurrence. It also may help reduce the extent of the lesions before surgery and facilitate the subsequent surgery. Most women with ovarian cancer have several cycles (an average of six) of chemotherapy as an outpatient, with rest periods in between. Moreover we must not forget that chemotherapy has side effects such as tiredness, hair loss and lowered immunity, although some can be controlled with suitable drugs. The most commonly used drug is carboplatin|, which may be given with paclitaxel (Taxol). Some other drugs that are less commonly used, or may be used if the cancer comes back, are: topotecan, liposomal doxorubicin, cisplatin, docetaxel, gemcitabine and etoposide. Finally, a correct anatomical definition of both nature and grading together with the evolution of the malignant disease is compulsory for an appropriate management and therapy [12].

Such a case is very interesting for the discrepancy between slight symptoms and severity of the disease, the solution of which was very complex requiring a skillful polyspecialized oncological team.

\section{Acknowlegements}

Special thanks to Salvatore Sciaretta (English Professor, Catania University) and Dott. Andreea Olteanu (Translator and interpreter).

\section{References}

[1] Halperin R, Zehavi S, Langer R, Hadas E, Bukovsky I, et al. (2001) Primary peritoneal serous papillary carcinoma: a new epidemiologic trend? A matched-case comparison with ovarian serous papillary cancer. Int J Gynecol Cancer 11:403-408.

[2] Piura B, Rabinovich A, Yanai-Inbar I (2001) Three primary malignancies related to BRCA mutation successively occurring in a BRCA1 185delAG mutation carrier. Eur J Obstet Gynecol Reprod Biol 97:241-244.

[3] Levine DA, Argenta PA, Yee CJ, Marshall DS, Olvera N, et al. (2003) Fallopian tube and primary peritoneal carcinomas associated with BRCA mutations. J Clin Oncol 21:4222-4227.

[4] Bloss JD, Liao SY, Buller RE, Manetta A, Berman ML, et al. (1993) Extraovarian peritoneal serous papillary carcinoma: a case-control retrospective comparison to papillary adenocarcinoma of the ovary. Gynecol Oncol 50:347-351. 
[5] Chen LM, Yamada SD, Fu YS, Baldwin RL, Karlan BY (2003) Molecular similarities between primary peritoneal and primary ovarian carcino- mas. Int J Gynecol Cancer 13:749-755.

[6] Kurman RJ, Shih IeM (2008) Pathogenesis of ovarian cancer: lessons from morphology and molecular biology and their clinical implications. Int J Gynecol Pathol 27:151-160.

[7] Jarboe EA, Folkins AK, Drapkin R, Ince TA, Agoston ES, et al. (2008) Tubal and ovarian pathways to pelvic epithelial cancer: a pathological perspective. Histopathology 53:127-138.

[8] Kindelberger DW, Lee Y, Miron A, Hirsch MS, Feltmate C, et al. (2007) Intraepithelial carcinoma of the fimbria and pelvic serous carcinoma: Evidence for a causal relationship. Am J Surg Pathol 31:161-169.

[9] Goodman MT, Correa CN, Tung KH, Roffers SD, Cheng Wu X, et al. (2003) Stage at diagnosis of ovarian cancer in the United States, 1992-1997. Cancer 97: 2648-2659.

10] Bloss JD, Brady MF, Liao SY, Rocereto T, Partridge EE, et al. (2003) Extraovarian peritoneal serous papillary carcinoma: a phase II trial of cisplatin and cyclophosphamide with comparison to a cohort with papillary serous ovarian carcinoma-a Gynecologic Oncology Group Study. Gynecol Oncol 89:148-54.

[11] Medeiros F, Muto MG, Lee Y, Elvin JA, Callahan MJ, et al. (2006) The tubal fimbria is a preferred site for early adenocarcinoma in women with familial ovarian cancer syndrome. Am J Surg Pathol 30:230236.

[12] Leanza V, Marilli I, Genovese F, Leanza G (2012) Uterine Myxoid Leiomyosarcoma Associated with Multiple Myomas in a Fertile Woman: A Case Report. Gynecol Obstet 2:128. doi:10.4172/21610932.1000128. 